\title{
FULLY TRANSITIVITY OF DIRECT SUMS AND DIRECT PRODUCTS OF QTAG-MODULES
}

\author{
AYAZUL HASAN $^{1, *}$, RAFIQUDDIN ${ }^{2}$ \\ ${ }^{1}$ Department of Mathematics, Faculty of Science, \\ Jazan University, Jazan- P.O. Box 2097, Kingdom of Saudi Arabia \\ ${ }^{2}$ Department of Applied Mathematics, Faculty of Engineering and Technology, \\ Aligarh Muslim University, Aligarh-202002, India \\ *Corresponding author: ayaz.maths@gmail.com \\ Received Sept. 24, 2018
}

\begin{abstract}
Aвstract. In this paper, a criterion for fully transitivity of direct sums of $Q T A G$-modules is established and sufficient conditions for fully transitivity of direct products of arbitrary $h$-reduced $Q T A G$-modules are presented. We also discuss some interesting properties of closed modules and consequently investigate influence of fully transitivity on separability and total projectivity of modules.

2010 Mathematics Subject Classification. 16K20.
\end{abstract}

Key words and phrases. QTAG-modules; fully transitive modules; fully transitive system of modules.

\section{Introduction and Preliminary Terminology}

Let $R$ be any ring. Consider the following two conditions on a module $M_{R}$ :

(I) Every finitely generated submodule of any homomorphic image of $M$ is a direct sum of uniserial modules.

(II) Given any two uniserial submodules $U$ and $V$ of a homomorphic image of $M$, for any submodule $W$ of $U$, any non-zero homomorphism $f: W \rightarrow V$ can be extended to a homomorphism $g: U \rightarrow V$, provided the composition length $d(U / W) \leq d(V / f(W))$.

A module $M_{R}$ satisfying $(I)$ and $(I I)$ is called a $T A G$-module, and a module satisfying only condition $(I)$ is called a $Q T A G$-module. The study of $Q T A G$-modules was initiated by Singh [10]. This is a very fascinating structure that has been the subject of research of many authors. Different notions and structures of $Q T A G$-modules have been studied, and a theory was developed, introducing several notions, interesting properties, and different characterizations of submodules. Many interesting results have been obtained, but there is still a lot to explore.

Let all rings discussed here be associative with unity $(1 \neq 0)$ and modules are unital $Q T A G$-modules. A module in which the lattice of its submodule is totally ordered is called a serial module; in addition if it has finite composition length it is called a uniserial module. An element $x \in M$ is uniform, if $x R$ is a non-zero uniform (hence uniserial) module and for any $R$-module $M$ with a unique decomposition series, $d(M)$ denotes its decomposition length.

DOI: $10.28924 / \mathrm{APJM} / 6-5$

(2019 Asia Pacific Journal of Mathematics 
For a uniform element $x \in M, e(x)=d(x R)$ and $H_{M}(x)=\sup \left\{d\left(\frac{y R}{x R}\right): y \in M, x \in y R\right.$ and $y$ uniform $\}$ are the exponent and height of $x$ in $M$, respectively. $H_{n}(M)$ denotes the submodule of $M$ generated by the elements of height at least $n$ and $H^{n}(M)$ is the submodule of $M$ generated by the elements of exponents at most $n$. The module $M$ is $h$-divisible if $M=M^{1}=\bigcap_{n=0}^{\infty} H_{n}(M)$ and it is $h$-reduced if it does not contain any $h$-divisible submodule. In other words it is free from the elements of infinite height. The module $M$ is called separable if every finitely generated submodule of $M$ can be embedded in a summand of $M$. A submodule $N \subset M$ is nice in $M$, if $H_{\sigma}(M / N)=\left(H_{\sigma}(M)+N\right) / N$ for all ordinals $\sigma$, i.e. every coset of $M$ modulo $N$ may be represented by an element of the same height.

The submodules $H_{n}(M), n \geq 0$ form a neighborhood system of zero, thus a topology known as $h$-topology arises. Closed modules [6] are also closed with respect to this topology. Thus, the closure of $N \subseteq M$ is defined as $\bar{N}=\bigcap_{n=0}^{\infty}\left(N+H_{n}(M)\right)$. Therefore, the submodule $N \subseteq M$ is closed with respect to $h$-topology if $\bar{N}=N$.

A family of nice submodules $\mathcal{N}$ of $M$ is called a nice system in $M$ if

(i) $0 \in \mathcal{N}$;

(ii) if $\left\{N_{i}\right\}_{i \in I}$ is any subset of $\mathcal{N}$, then $\sum_{i \in I} N_{i} \in \mathcal{N}$;

(iii) given any $N \in \mathcal{N}$ and any countable subset $X$ of $M$, there exists $K \in \mathcal{N}$ containing $N \cup X$, such that $K / N$ is countably generated [5].

An $h$-reduced module $M$ is called totally projective if it has a nice system.

It is interesting to note that almost all the results which hold for $T A G$-modules are also valid for $Q T A G$ modules [8]. In what follows, all notations and notions are standard and will be in agreement with those used in $[1,2]$.

\section{Main Concepts and Results}

We start here with a recollection of the following notions from [7].

Definition 2.1. The generalized height of $x$ in $M$ denoted by

$$
H_{M}^{*}(x)=\left\{\begin{array}{l}
\alpha, \text { if } x \neq 0 \text { and } \alpha+1 \text { is the first ordinal such that } x \notin H_{\alpha+1}(M) \\
\infty, \text { if } x=0 .
\end{array}\right.
$$

For any uniform element $x \in M$, there exist uniform elements $x_{1}, x_{2}, \ldots$ such that $x R \supseteq x_{1} R \supseteq x_{2} R \supseteq \ldots$ and $d\left(\frac{x_{i} R}{x_{i+1} R}\right)=1$. Now the $U l m$-sequence of $x$ is defined as $U(x)=\left(H(x), H\left(x_{1}\right), H\left(x_{2}\right), \ldots\right)$. This is analogus to the $U$-sequences in groups [1]. These sequences are partially ordered because $U(x) \leq U(y)$ if $H\left(x_{i}\right) \leq H\left(y_{i}\right)$ for every $i$.

With the aid of the above discussion we are able to define the following. 
Definition 2.2. For any element $x$ of the $Q T A G$-module $M$, we denote by $[\mathbb{H}]_{(x)}$ the height matrix:

$$
[\mathbb{H}]_{(x)}=\left(\begin{array}{c}
H_{M}^{*}(x) H_{M}^{*}\left(x_{1}\right) \cdots H_{M}^{*}\left(x_{k}\right) \cdots \\
\ldots \ldots \ldots \ldots \ldots \ldots \ldots \ldots \ldots \\
H_{M}^{*}\left(x_{n}\right) H_{M}^{*}\left(x_{n_{1}}\right) \cdots H_{M}^{*}\left(x_{n_{k}}\right) \cdots \\
\ldots \ldots \ldots \ldots \ldots \ldots \ldots \ldots \ldots \ldots
\end{array}\right) .
$$

Such a matrix can be interpreted as a function $f: \mathbb{N} \times \mathbb{N} \rightarrow \mathcal{O}_{n} \cup\{\infty\}$ (where $\mathcal{O} n$ is the class of all ordinals) such that $f(i, j)=\alpha_{i j}$, where

$$
\left(\alpha_{i j}\right)=\left(\begin{array}{c}
\alpha_{11} \alpha_{12} \cdots \alpha_{1 n} \cdots \\
\ldots \ldots \ldots \ldots \ldots \\
\alpha_{n 1} \alpha_{n 2} \cdots \alpha_{n n} \cdots \\
\ldots \ldots \ldots \ldots \ldots
\end{array}\right) .
$$

The class of all height matrices is denoted by $\mathcal{H}$. Let $A, B \in \mathcal{H}, A=\left(\alpha_{i j}\right), B=\left(\beta_{i j}\right)$, then we say that $A \leq B$ if $\alpha_{i j} \leq \beta_{i j}$ for all $i, j \in \mathbb{N}$. The set of all height matrices is denoted by $\inf _{\mathcal{H}}$.

Fully transitivity is one of interesting properties of $Q T A G$-modules. Following [9], a $Q T A G$-module $M$ is fully transitive if for $x, y \in M, U(x) \leq U(y)$, there is an endomorphism $\phi$ of $M$ such that $\phi(x)=\phi(y)$. Here we study these modules in the light of height matrices.

Definition 2.3. A QTAG-module $M$ is said to be fully transitive if for all elements $x, y \in M$ such that $[\mathbb{H}]_{(x)} \leq[\mathbb{H}]_{(y)}$ there exists an endomorphism $\phi$ of $M$ with the property $\phi(x)=y$.

As a consequence, we have the following.

Proposition 2.1. For an $h$-reduced $Q T A G$-module $M$, the following are equivalent:

(i) for all $x, y \in M$, the condition $[\mathbb{H}]_{(x)} \leq[\mathbb{H}]_{(y)}$ and $d\left(\frac{x R}{y R}\right)=1$, implies existence of an endomorphism $\phi$ of $M$ such that $\phi(x)=y$;

(ii) for all $x, y \in M$, the condition $[\mathbb{H}]_{(x)} \leq[\mathbb{H}]_{(y)}$, implies existence of an endomorphism $\phi$ of $M$ such that $\phi(x)=y$.

We are now ready to prove the following theorem.

Theorem 2.1. Let $M$ be a $Q T A G$-module. Then $M$ is fully transitive if and only if its h-reduced submodule is fully transitive.

Proof. Suppose $M$ is a fully transitive $Q T A G$-module, fully transitivity of its $h$-reduced submodule easily follows from the definition.

Conversely, Let $M=L \oplus K$, where $L$ is the $h$-reduced submodule of $M, K$ is its $h$-divisible submodule. Consider arbitrary non-zero elements $x, y \in M$ such that $[\mathbb{H}]_{(x)} \leq[\mathbb{H}]_{(y)}$ and $d\left(\frac{x R}{y R}\right)=1$. Let $x=a+b, y=c+d$. Then we have four cases to consider:

Case (i): $a \neq 0, c \neq 0$, where $a, c \in L ; b, d \in K$. Then $[\mathbb{H}]_{(x)}=[\mathbb{H}]_{(a)},[\mathbb{H}]_{(y)}=[\mathbb{H}]_{(c)}$ and $[\mathbb{H}]_{(a)} \leq[\mathbb{H}]_{(c)}$. So, there is an endomorphism $\phi$ of $M$ such that $\phi(a)=c$ follows from fully transitivity of $L$. Now we can extend $\phi$ to an endomorphism $\psi$ of $M$ such that $\psi(z)=\phi(z)$ if $z \in L$, and $\psi(z)=0$ if $z \in K$. 
Case (ii): $a \neq 0, c=0$. Consider the submodule $x R \subset M$. Construct a mapping $\varphi: x R \rightarrow K$ such that $\varphi(x)=y$. It is easy to verify that $\varphi$ is a homomorphism. Let $\theta: x R \rightarrow M$ be an embedding. Since $K$ is an injective module, there exist a homomorphism $\sigma: M \rightarrow K$ such that $\sigma(\theta)=\varphi$ i.e., $\sigma(x)=y$.

Case (iii): $a=0, c \neq 0$. This is not possible.

Case (iv): $a=0, c=0$ i.e., $x, y \in K$ and $d\left(\frac{x R}{y R}\right)=1$. Consider the submodule $x R, y R$ from $K$. There exists a homomorphism $\rho: x R \rightarrow y R$ such that $\rho(x)=y$. Let $\vartheta: x R \rightarrow K$ be an embedding. Since $K$ is injective module, there exists an endomorphism $\eta$ of $K$ such that $\eta(\vartheta)=\rho$, i.e., $\eta(x)=y$. The endomorphism $\eta$ evidently can be extended to an endomorphism of $M$. Thus, $M$ is fully transitive.

We give two main concepts which will be used in studying fully transitivity of direct sums and direct products. The first of them appeared in the work of Hasan at el. [4] with a different perspective.

Definition 2.4. A system of $Q T A G$-modules $\left\{M_{i}\right\}_{i \in I}$ is said to be fully transitive if for each pair of $Q T A G$-modules $\left(M_{i}, M_{j}\right)_{i, j \in I}(i$ can coincide with $j)$ and for every $x \in M_{i}, y \in M_{j}$ satisfying $[\mathbb{H}]_{(x)} \leq[\mathbb{H}]_{(y)}$, there exists a homomorphism $\phi$ form $M_{i}$ to $M_{j}$ such that $\phi(x)=y$.

Definition 2.5. We say that a system of $Q T A G$-modules $\left\{M_{i}\right\}_{i \in I}$ satisfies the monotonicity condition for height matrices if for each $Q T A G$-module $M_{j}$ and each element $0 \neq x_{j} \in M_{j}(j \in I)$ the relations

(i) $\inf _{\mathcal{H}}\left\{[\mathbb{H}]_{\left(x_{i_{1}}\right)}, \ldots,[\mathbb{H}]_{\left(x_{i_{s}}\right)}\right\} \leq[\mathbb{H}]_{\left(x_{j}\right)}$, where $x_{i_{k}} \in M_{i_{k}}, i_{k} \in I, k=\overline{1, s}, i_{k} \neq i_{m}, k \neq m$;

(ii) $[\mathbb{H}]_{\left(x_{j}\right)} \geq[\mathbb{H}]_{\left(x_{k}\right)}$ for all $k=\overline{1, s}$,

imply existence of the elements $x_{j_{1}}, \ldots, x_{j_{r}} \in M_{j}$ with the following properties:

$(i)^{\prime} x_{j_{1}}+\cdots+x_{j_{r}}=x_{j} ;$

$(\text { ii })^{\prime}$ for each element $x_{j_{m}}(m=\overline{1, r})$ there exists an element $x_{i_{k}}(k=\overline{1, s})$ such that $[\mathbb{H}]_{\left(x_{j_{m}}\right)} \geq[\mathbb{H}]_{\left(x_{i_{k}}\right)}$.

Remark 2.1. If a system of $Q T A G$-modules is fully transitive, each its subsystem is also fully transitive. If a system of QT AG-modules satisfies the monotonicity condition for height matrices, each its subsystem also satisfies the condition for height matrices.

Theorem 2.2. Let $M$ be a $Q T A G$-module such that $M=\bigoplus_{i \in I} M_{i}$. Then $M$ is fully transitive if and only if the system $\left\{M_{i}\right\}_{i \in I}$ is fully transitive and satisfies the monotonicity condition for height matrices.

Proof. Let $M$ be a fully transitive $Q T A G$-module. Consider arbitrary modules $M_{\alpha}, M_{\beta} \in\left\{M_{i}\right\}_{i \in I}$ and elements $x_{\alpha} \in M_{\alpha}, x_{\beta} \in M_{\beta}$ such that $[\mathbb{H}]_{\left(x_{\alpha}\right)} \leq[\mathbb{H}]_{\left(x_{\beta}\right)}$. Then $[\mathbb{H}]_{\left(\theta_{\alpha} x_{\alpha}\right)} \leq[\mathbb{H}]_{\left(\theta_{\beta} x_{\beta}\right)}$, where $\theta_{\alpha}: M_{\alpha} \rightarrow M, \theta_{\beta}: M_{\beta} \rightarrow M$, are embeddings. Fully transitivity of $M$ implies existence of endomorphism $\phi$ of $M$ such that $\left(\phi \theta_{\alpha}\right)\left(x_{\alpha}\right)=\theta_{\beta}\left(x_{\beta}\right)$ and therefore, $\left(\pi_{\beta} \phi \theta_{\alpha}\right)\left(x_{\alpha}\right)=x_{\beta}$, where $\pi_{\beta}: M \rightarrow M_{\beta}$ is a projection.

Let's demonstrate that the system $\left\{M_{i}\right\}_{i \in I}$ satisfies the monotonicity condition for height matrices. Consider an arbitrary module $M_{j} \in\left\{M_{i}\right\}_{i \in I}$ and an element $0 \neq x_{j} \in M_{j}$ such that $\inf _{\mathcal{H}}\left\{[\mathbb{H}]_{\left(x_{i_{1}}\right)}, \ldots,[\mathbb{H}]_{\left(x_{i_{s}}\right)}\right\} \leq[\mathbb{H}]_{\left(x_{j}\right)}$, $[\mathbb{H}]_{\left(x_{j}\right)} \geq[\mathbb{H}]_{\left(x_{k}\right)}$ for all $k=\overline{1, s}$. For each $k=\overline{1, s}$, consider embeddings $\theta_{i_{k}}: M_{i_{k}} \rightarrow M$ and $\theta_{j}: M_{j} \rightarrow M$, where $M_{i_{k}} \in\left\{M_{i}\right\}_{i \in I}$ and $x_{i_{k}} \in M_{i_{k}}$. Then $[\mathbb{H}]_{\left(\theta_{i_{1}} x_{i_{1}}+\cdots+\theta_{i_{s}} x_{i_{s}}\right)} \leq[\mathbb{H}]_{\left(\theta_{j} x_{j}\right)}$, and fully transitivity of $M$ implies existence of endomorphism $\phi$ of $M$ such that $\theta_{j}\left(x_{j}\right)=\phi\left(\theta_{i_{1}} x_{i_{1}}+\cdots+\theta_{i_{s}} x_{i_{s}}\right)=\left(\phi \theta_{i_{1}}\right) x_{i_{1}}+\cdots+\left(\phi \theta_{i_{s}}\right) x_{i_{s}}$. We have $x_{j}=\left(\pi_{j} \theta_{j}\right)\left(x_{j}\right)=\left(\pi_{j} \phi \theta_{i_{1}}\right)\left(x_{i_{1}}\right)+\cdots+\left(\pi_{j} \phi \theta_{i_{s}}\right)\left(x_{i_{s}}\right)$, where $\pi_{j}: M \rightarrow M_{j}$ is a projection. Let $x_{j_{k}}=\left(\pi_{j} \phi \theta_{i_{k}}\right)\left(x_{i_{k}}\right)$ 
for all $k=\overline{1, s}$. Then $x_{j}=x_{j_{1}}+\cdots+x_{j_{s}}$, and for each element $x_{j_{k}}(k=\overline{1, s})$ there exists an element $x_{i_{k}}(k=\overline{1, s})$ such that $[\mathbb{H}]_{\left(x_{i_{k}}\right)} \leq[\mathbb{H}]_{\left(x_{j_{k}}\right)}$.

Conversely, let $x, y \in M$, and $[\mathbb{H}]_{(x)} \leq[\mathbb{H}]_{(y)}$. Let $x=x_{i_{1}}+\cdots+x_{i_{r}}, y=y_{m_{1}}+\cdots+y_{m_{n}}$, where $x_{i_{t}} \in M_{i_{t}}$, $y_{m_{j}} \in M_{m_{j}}, i_{t}, m_{j} \in I$ for all $t=\overline{1, r}$ and $j=\overline{1, n}$. Let's show that for each element $y_{m_{j}}(j=\overline{1, n})$ there exists a homomorphism $\phi_{j}: M \rightarrow M_{m_{j}}$ such that $\phi_{j}(x)=y_{m_{j}}$. Then $y=\left(\phi_{j_{1}}+\cdots+\phi_{j_{n}}\right)(x)$ and $\left(\phi_{j_{1}}+\cdots+\phi_{j_{n}}\right)$ is an endomorphism of $M$. If for the element $y_{m_{j}}$ there exists an element $x_{i_{k}}$ such that $[\mathbb{H}]_{\left(y_{m_{j}}\right)} \geq[\mathbb{H}]_{\left(x_{i_{k}}\right)}$, existence of a homomorphism $\psi_{j}: M_{i_{k}} \rightarrow M_{m_{j}}$ with the property $\psi_{j}\left(x_{i_{k}}\right)=y_{m_{j}}$ follows from fully transitivity of the system $\left\{M_{i}\right\}_{i \in I}$. Then we put $\phi_{j}(x)=\psi_{j}\left(x_{i_{k}}\right)$, i.e., $\phi_{j}=\psi_{j} \pi_{i_{k}}$, where $\pi_{i_{k}}: M \rightarrow M_{i_{k}}$ is a projection. Let there be no element $x_{i_{k}}$ with the property $[\mathbb{H}]_{\left(y_{m_{j}}\right)} \geq[\mathbb{H}]_{\left(x_{i_{k}}\right)}$. Since $[\mathbb{H}]_{\left(y_{m_{j}}\right)} \geq \inf _{\mathcal{H}}\left\{[\mathbb{H}]_{\left(x_{i_{k}}\right)}\right\}_{k=\overline{1, r}}$, and the system $\left\{M_{i}\right\}_{i \in I}$ satisfies the monotonicity condition for height matrices, there exist elements $y_{m_{j_{u}}} \in M_{m_{j}}(u=\overline{1, t})$ such that $y_{m_{j}}=y_{m_{j_{1}}}+\cdots+y_{m_{j_{t}}}$ and for each $y_{m_{j_{u}}}(u=\overline{1, t})$ one can find $x_{i_{k_{u}}} \in\left\{x_{i_{k}}\right\}_{k=\overline{1, r}}$ such that $[\mathbb{H}]_{\left(y_{m_{j_{u}}}\right)} \geq[\mathbb{H}]_{\left(x_{i_{k_{u}}}\right)}$. Then fully transitive of the system $\left\{M_{i}\right\}_{i \in I}$ implies existence of a homomorphism $\psi_{j_{u}}: M_{i_{k_{u}}} \rightarrow M_{m_{j}}$ such that $\psi_{j_{u}}\left(x_{i_{k_{u}}}\right)=y_{m_{j_{u}}}$, and $\psi_{j_{1}}\left(x_{i_{k_{1}}}\right)+\cdots+\psi_{j_{t}}\left(x_{i_{k_{t}}}\right)=y_{m_{j}}$. Then $\phi_{j}(x)=\psi_{j_{1}}\left(x_{i_{k_{1}}}\right)+\cdots+\psi_{j_{t}}\left(x_{i_{k_{t}}}\right)$, where $\phi_{j}=\psi_{j_{1}} \pi_{i_{k_{1}}}+\cdots+\psi_{j_{t}} \pi_{i_{k_{t}}}, \pi_{i_{k_{u}}}: M \rightarrow M_{i_{k_{u}}}$ are projections. Thus $\phi_{j}(x)=b_{m_{j}}$.

The following proposition demonstrates that fully transitivity and fulfilment of the monotonicity condition for height matrices for the system of $Q T A G$-modules $\left\{M_{i}\right\}_{i \in I}$ are necessary for fully transitivity of a direct product of arbitrary $Q T A G$-modules.

Proposition 2.2. Let $M$ be a $Q T A G$-module such that $M=\prod_{i \in I} M_{i}$ is fully transitive. Then the system $\left\{M_{i}\right\}_{i \in I}$ is fully transitive and satisfies the condition of monotonicity for height matrices.

Proof. The proof of this statement is similar to proof of necessity in the previous theorem.

Definition 2.6. We say that the system of the QTAG-modules $\left\{M_{i}\right\}_{i \in I}$ satisfies the condition of h-finiteness for height matrices, if for each $Q T A G$-module $M_{j} \in\left\{M_{i}\right\}_{i \in I}$ and each element $0 \neq x_{j} \in M_{j}$ such that $e\left(x_{j}\right)=\infty$ the conditions

$$
[\mathbb{H}]_{\left(x_{j}\right)} \geq \inf _{\mathcal{H}}\left\{[\mathbb{H}]_{\left(x_{\alpha}\right)}\right\}_{\alpha \in I}
$$

imply existence of a finite subsystem of elements $\left\{x_{\alpha_{k}}\right\}_{\alpha_{k} \in I, k=\overline{1, n}}$ such that

$$
[\mathbb{H}]_{\left(x_{j}\right)} \geq \inf _{\mathcal{H}}\left\{[\mathbb{H}]_{\left(x_{\alpha_{k}}\right)}\right\}_{\alpha_{k} \in I, k=\overline{1, n}}
$$

Proposition 2.3. Let $M$ be a $Q T A G$-module such that $M=\prod_{i \in I} M_{i}$. If the system $\left\{M_{i}\right\}_{i \in I}$ is fully transitive and satisfies the conditions of monotonicity and h-finiteness for height matrices, then $M$ is fully transitive.

Proof. Let $x, y \in M$ such that $[\mathbb{H}]_{(x)} \leq[\mathbb{H}]_{(y)}$, where $x=\left(\ldots, x_{i}, \ldots\right), y=\left(\ldots, y_{i}, \ldots\right)$. Consider an arbitrary coordinate $y_{j}$ of the element $y$ there exists a finite subsystem of coordinates $\left\{x_{i_{u}}\right\}_{u=\overline{1, n}}$ of the element $x$ such that $[\mathbb{H}]_{\left(y_{j}\right)} \geq \inf _{\mathcal{H}}\left\{[\mathbb{H}]_{\left(x_{i_{u}}\right)}\right\}_{u=\overline{1, n}}$.

We have two cases to consider: $(i) e\left(y_{j}\right)<\infty,(i i) e\left(y_{j}\right)=\infty$, here we assume that almost all the coordinates $x_{i}$ of the element $x$ are different from zero. Let $e\left(y_{j}\right)<\infty$. Since the height matrix $[\mathbb{H}]_{\left(y_{j}\right)}$ contains a finite number $\gamma_{n_{k}}$ of ordinals different from $\infty$ and $[\mathbb{H}]_{\left(y_{j}\right)} \geq \inf _{\mathcal{H}}\left\{[\mathbb{H}]_{\left(x_{i}\right)}\right\}_{i \in I}$, there exists a finite subset $\left\{x_{i_{u}}\right\}_{u=\overline{1, n}} \subset\left\{x_{i}\right\}_{i \in I}$ such that $[\mathbb{H}]_{\left(y_{j}\right)} \geq \inf _{\mathcal{H}}\left\{[\mathbb{H}]_{\left(x_{i_{u}}\right)}\right\}_{u=\overline{1, n}}$. If $e\left(y_{j}\right)=\infty$, then, taking into account the inequality $[\mathbb{H}]_{\left(y_{j}\right)} \geq[\mathbb{H}]_{(x)}$ 
and the fact that the height matrix of every element of a module consists of at most countable set of ordinals, we prove existence of the subset $\left\{x_{v}\right\}_{v \in I} \subseteq\left\{x_{i}\right\}_{i \in I}$ such that $[\mathbb{H}]_{\left(y_{j}\right)} \geq \inf _{\mathcal{H}}\left\{[\mathbb{H}]_{\left(x_{v}\right)}\right\}_{v \in I}$. Since the system $\left\{M_{i}\right\}_{i \in I}$ satisfies the condition of $h$-finiteness, there exists a finite subset $\left\{x_{v_{u}}\right\}_{u=\overline{1, m}} \subseteq\left\{x_{v}\right\}_{v \in I}$ such that $[\mathbb{H}]_{\left(y_{j}\right)} \geq \inf _{\mathcal{H}}\left\{[\mathbb{H}]_{\left(x_{v_{u}}\right)}\right\}_{u=\overline{1, m}}$.

Let's demonstrate that there exists an endomorphism $\phi$ of $M$ such that $\phi(x)=y$. To do this, it is sufficient to see that for an arbitrary coordinate $y_{j}$ of the element $y$ there exists a homomorphism $\phi_{j}: M \rightarrow M_{j}$ such that $\phi_{j}(x)=y_{j}$. Let $[\mathbb{H}]_{\left(y_{j}\right)} \geq \inf _{\mathcal{H}}\left\{[\mathbb{H}]_{\left(x_{i_{k}}\right)}\right\}_{i_{k} \in I, k=\overline{1, n}}$. where $y_{j} \in M_{j}, x_{i_{k}} \in M_{i_{k}}$. Since the system $\left\{M_{i}\right\}_{i \in I}$ is fully transitive and satisfies the condition of monotonicity for height matrices, the subsystem $\left\{M_{j}, M_{i_{k}}\right\}_{k=1, n}$ where $M_{j} \neq M_{i_{k}}$ for all $k=\overline{1, n}$, is also fully transitive and satisfies the condition of monotonicity for height matrices. Therefore, the $Q T A G$-module $M^{\prime}=M_{j} \oplus \oplus_{k=1}^{n} M_{i_{k}}$, as it follows from Theorem 2.2, is fully transitive. So there exists a homomorphism $\phi_{j}^{\prime}: M^{\prime} \rightarrow M_{j}$ such that $\left(\phi_{j}^{\prime} \theta\right)\left(x_{i_{1}}+\cdots+x_{i_{n}}\right)=y_{j}$, where $\theta: \oplus_{k=1}^{n} M_{i_{k}} \rightarrow$ $M^{\prime}$ is an embedding, $\phi_{j}^{\prime}=\pi_{j} \phi_{j}^{\prime \prime}, \pi_{j}: M^{\prime} \rightarrow M_{j}$ is a projection, and $\phi_{j}^{\prime \prime}$ is an endomorphism of $M^{\prime}$, where $\left(\phi_{j}^{\prime \prime} \theta\right)\left(x_{i_{1}}+\cdots+x_{i_{n}}\right)=\theta_{j}\left(y_{j}\right), \theta_{j}: M_{j} \rightarrow M^{\prime}$ is an embedding. Since $M^{\prime}$ is a direct summand of $M$, one can extend the homomorphism $\phi_{j}^{\prime} \theta$ to a homomorphism $\phi_{j}: M \rightarrow M_{j}$, mapping $x$ to the element $y_{j}$. And if the $Q T A G$-module $M_{j}$ coincide with one of the $Q T A G$-modules $M_{i_{k}}, k=\overline{1, n}$, similar reasoning for the subsystem $\left\{M_{i_{k}}\right\}_{k=\overline{1, n}}$, yield the homomorphism $\phi_{j}: M \rightarrow M_{j}$ which maps the element $x$ to the element $y_{j}$.

Now we present here some interesting properties of closed modules. First, we prove the following working lemma.

Lemma 2.1. A system of closed QTAG-modules $\left\{M_{i}\right\}_{i \in I}$ satisfies the condition of monotonicity for height matrices if and only if the system $\left\{M_{i}^{\lambda}\right\}_{i \in I}$ (where $M_{i}^{\lambda}$ are the component of $M_{i}$ ) satisfies the condition of monotonicity for height matrices.

Proof. Necessity directly follows from the definition of monotonicity of a system. Let's prove sufficiency. Consider an arbitrary $Q T A G$-module $M_{j} \in\left\{M_{i}\right\}_{i \in I}$ and an arbitrary element $x_{j} \in M_{j}$ such that $[\mathbb{H}]_{\left(x_{j}\right)} \geq$ $\inf _{\mathcal{H}}\left\{[\mathbb{H}]_{\left(x_{i_{k}}\right)}\right\}_{i_{k} \in I, k=\overline{1, n}}$. Here $x_{i_{u}} \neq x_{i_{s}}$ if $i_{u} \neq i_{s}$. Let $[\mathbb{H}]_{\left(x_{j}\right)} \geq[\mathbb{H}]_{\left(x_{i_{k}}\right)}$ for any $k=\overline{1, n}$. Let's consider

$$
x_{j}=x_{j_{1}}+\cdots+x_{j_{r}} x_{i_{k}}=x_{i_{k}}^{(1)}+\cdots+x_{i_{k}}^{(m)}(k=\overline{1, n}) .
$$

Then

$$
[\mathbb{H}]_{\left(x_{j_{\beta}}\right)} \geq[\mathbb{H}]_{\left(x_{j}\right)} \geq \inf _{\mathcal{H}}\left\{[\mathbb{H}]_{\left(x_{i_{k}}\right)}\right\}_{i_{k} \in I, k=\overline{1, n}}=\inf _{\mathcal{H}}\left\{[\mathbb{H}]_{\left(x_{i_{k}}^{(\gamma)}\right)}\right\}_{i_{k} \in I, k=\overline{1, n}, \gamma=\overline{1, m}}
$$

for each $\beta=\overline{1, r}$. Consider the element $x_{j_{\beta}}(\beta=\overline{1, r})$ such that $[\mathbb{H}]_{\left(x_{j_{\beta}}\right)} \geq[\mathbb{H}]_{\left(x_{i_{k}}^{(\gamma)}\right)}$ for all $\gamma=\overline{1, m}$ for any $k=\overline{1, n}$ (if there are no such elements, the condition of monotonicity for height matrices is satisfied trivially). Then, for each element $x_{j_{\beta}}$, one can find elements

$$
\left.\left\{a_{\eta}^{(\beta)}\right\}_{\eta=\overline{1, s}} \subseteq\left(x_{i_{k}}^{(\gamma)}\right)\right\}_{i_{k} \in I, k=\overline{1, n}, \gamma=\overline{1, m}} .
$$

Here $[\mathbb{H}]_{\left(x_{j_{\beta}}\right)} \geq \inf _{\mathcal{H}}\left\{[\mathbb{H}]_{\left(a_{\eta}^{(\beta)}\right)}\right\}_{\eta=\overline{1, s}}$. Since the system $\left\{M_{i}^{\lambda}\right\}_{i \in I}$ satisfies the condition of monotonicity for height matrices, there exist elements $b_{j_{1}}^{(\beta)}, \ldots, b_{j_{t}}^{(\beta)}$ such that

$$
b_{j_{1}}^{(\beta)}+\cdots+b_{j_{t}}^{(\beta)}=x_{j_{\beta}},
$$


and for each element $b_{j_{\delta}}^{(\beta)}(\delta=\overline{1, t})$ there exists $a_{\eta}^{(\beta)}(\eta=\overline{1, s})$ such that $[\mathbb{H}]_{\left(b_{j_{\delta}}^{(\beta)}\right)} \geq[\mathbb{H}]_{\left(a_{\eta}^{(\beta)}\right)}$. Then $[\mathbb{H}]_{\left(b_{j_{\delta}}^{(\beta)}\right)} \geq$ $[\mathbb{H}]_{\left(x_{i_{k}}\right)}$ for a certain $k=\overline{1, n}$, because $a_{\eta}^{(\beta)}$ is one of the summands in the decomposition of the element $x_{i_{k}}$. Further, replacing the elements $x_{j_{\beta}}$ by their decomposition $b_{j_{1}}^{(\beta)}+\cdots+b_{j_{t}}^{(\beta)}$, we obtain a new decomposition for the element $x_{j}$, and for each element $c_{j_{\sigma}}$ from this decomposition one can find an element $x_{i_{k}}(k=\overline{1, n})$ such that $[\mathbb{H}]_{\left(c_{j_{\sigma}}\right)} \geq[\mathbb{H}]_{\left(x_{i_{k}}\right)}$.

The following proposition is of some interest.

Proposition 2.4. Any system of fully transitive closed $Q T A G$ - modules satisfies the condition of monotonicity for height matrices.

Proof. The proof follows from Lemma 2.1, [4, Proposition 2.1] and Theorem 2.2.

Returning to our main term, we have the following consequence of Proposition 2.3.

Corollary 2.1. Let $M$ be a $Q T A G$-module such that $M=\prod_{i \in I} M_{i}$, where $M_{i}(i \in I)$ are closed modules. Then $M$ is fully transitive if and only if the following conditions are satisfied:

(i) $\left\{M_{i}\right\}_{i \in I}$ is fully transitive system;

(ii) the system $\left\{M_{i}\right\}_{i \in I}$ satisfies the condition of monotonicity for height matrices.

We continue with other statement, namely:

Corollary 2.2. For the closed $Q T A G$-modules $M_{i}(i \in I)$, the following conditions are equivalent:

(i) $\prod_{i \in I} M_{i}$ is fully transitive;

(ii) $\bigoplus_{i \in I} M_{i}$ is fully transitive;

(iii) $\left\{M_{i}\right\}_{i \in I}$ is fully transitive system.

Proof. The proof follows from Proposition 2.4, Corollary 2.1 and Theorem 2.2.

We end this paper with the following corollary.

Corollary 2.3. If each of the $h$-reduced closed $Q T A G$-modules $M_{i}(i \in I)$ satisfies at least one of the following conditions:

(i) $M_{i}$ is separable;

(ii) $M_{i}$ is totally projective,

then $\prod_{i \in I} M_{i}$ and $\bigoplus_{i \in I} M_{i}$ are fully transitive.

Proof. By Lemma 2.1, one can consider that each $M_{i}(i \in I)$ is a $Q T A G$-module. Let's show that $\bigoplus_{i \in I} M_{i}$ is fully transitive. Let $I^{\prime}=\left\{i \in I \mid M_{i}\right.$ is separable $\}$, then $\bigoplus_{i \in I} M_{i}=\bigoplus_{i \in I^{\prime}} M_{i}=\bigoplus_{i \in I \backslash I^{\prime}} M_{i}$. Note that $\bigoplus_{i \in I^{\prime}} M_{i}$ is separable ( $\underset{i \in I \backslash I^{\prime}}{\bigoplus} M_{i}$ is totally projective) as a direct sum of separable (totally projective modules). Since each totally projective module is fully transitive [3], $\bigoplus_{i \in I \backslash I^{\prime}} M_{i}$ is fully transitive. Therefore, we obtain that $\bigoplus_{i \in I} M_{i}$ is fully transitive. Corollary 2.2 makes it possible to say that $\prod_{i \in I} M_{i}$ is fully transitive. 


\section{ACKNOWLEDGMENTS}

The authors would like to express their sincere thanks to the referee for his/her careful reading of the paper, and to the Editor, for his/her valuable editorial work.

\section{REFERENCES}

[1] Fuchs L., Infinite Abelian Groups, Vol. I, Academic Press, New York, 1970.

[2] Fuchs L., Infinite Abelian Groups, Vol. II, Academic Press, New York, 1973.

[3] Hasan A., Some results of transitivity for QTAG-modules, (Communicated).

[4] Hasan A., Sikander F. and Begum F., On Ulm support in QTAG-modules, Scientia Series A., Math. Sci., 25 (2014), 9-15.

[5] Mehdi A., Abbasi M. Y. and Mehdi F., On $(\omega+n)$-projective modules, Ganita Sandesh, 20(1) (2006), 27-32.

[6] Mehdi A. and Khan M.Z., On closed modules, Kyungpook Math. J., 24(1) (1984), 45-50.

[7] Mehdi A., Naji S.A.R.K. and Hasan A., Small homomorphisms and large submodules of QTAG-modules, Scientia Series A., Math. Sci., 23 (2012), 19-24.

[8] Mehran H. and Singh S., On $\sigma$-pure submodules of QTAG-modules, Arch. Math., 46 (1986), 501-510.

[9] Naji S.A.R.K., A study of different structures in QTAG-modules, Ph.D. thesis, Aligarh Muslim University, (2010).

[10] Singh S., Abelian groups like modules, Act. Math. Hung, 50 (1987), 85-95. 